\title{
Psychological Resilience, Hope, and Adaptability as Protective Factors in Times of Crisis: A Study in Greek and Cypriot Society During the Covid-19 Pandemic
}

\author{
Loucia Dimitriou*, Mirsiana Drakontaides, Demetris Hadjicharalambous \\ Psychology and Social Sciences Department, Frederick University, Limassol, Cyprus \\ E-mail: pre.dl@frederick.ac.cy
}

Received: 1 September 2020; Revised: 24 September 2020; Accepted: 12 October 2020

\begin{abstract}
Various studies have assessed resilience in relation to risk and protective factors during periods of crisis, and have shown that values such as hope and optimism are significantly related to peoples' level of quality of life in times of crisis. The Covid-19 pandemic has proven to be a great challenge as it is an unprecedented global health crisis. Our study aims to explore the effects of psychological resilience, hope, and adaptability when people experience real or perceived possible danger. The present study was quantitative, and it applied three different self-report questionnaires, namely the CD RISK to assess psychological resiliency (Connor \& Davidson, 2003), the AHS (Adult-Hope-Scale) (Snyder, 1994) to assess hope, and a third questionnaire, the CAQ (Covid-19-Adaptation-Questionnaire), to explore perceived ability to adapt to changes in daily life due to the imposed quarantine as well as trust in state policies and the media during the peak of the pandemic in Greece and Cyprus. Demographic data such as age, gender, place of residence, and level of education were collected by using a Personal Information Form. We administered the instruments to 205 Greek and Cypriot men and women in age groups of 18 years or older. Our findings showed that high scores in hope could predict higher levels of psychological resilience and the ability to adapt in the face of adversity. We have also found a significant positive relationship between age and level of education with psychological resiliency. Older participants and participants with higher levels of education showed higher levels of psychological resiliency and adaptation in comparison with the younger and less educated subjects. At the same time, findings indicated that although people adapted and complied with extreme social isolation measures, neither did they actually trust the relevant state policies, nor did they rely on mass media for information regarding the pandemic.
\end{abstract}

Keywords: adaptability, Covid-19, hope, optimism, pandemic, psychological resiliency

\section{Introduction}

\subsection{Background of the study}

Psychological Resilience is comprehensively defined as the ability of a dynamic system to adapt successfully to any disturbances that threaten its operation, to withstand or recover from significant challenges that threaten its stability, sustainability, and growth (Wright et al., 2013). The coronavirus Covid-19 pandemic has proven to be a defining global

Copyright (C2020 Loucia Dimitriou, et al

DOI: https://doi.org/10.37256/ser.212021618

This is an open-access article distributed under a CC BY license

(Creative Commons Attribution 4.0 International License)

https://creativecommons.org/licenses/by/4.0/ 
health crisis of our time and, reportedly, one of the greatest challenges that humanity has faced since World War Two. According to the United Nations Development Program (UNDP), the pandemic is much more than a health crisis; it's also an unprecedented socio-economic crisis. According to the UNDP report (2020), Covid-19 seems to be creating pressures to every one of the countries it affects, since it has the potential to bring forth social, economic and political effects that will have a longstanding impact (UNDP, 2020). Since its emergence in Asia late last year, the virus has spread to every continent except Antarctica. The World Health Organization (WHO) declared Covid-19 as a public health emergency of international concern (Carpio Carmen, 2020) on 30th January 2020, and on 11th March, it was declared as a pandemic (Emanuel et al., 2020; Felix et al., 2020). These grave and rapid developments have created chaos all over the world as isolation, contact restrictions, and economic shutdown imposed a complete change to the psychosocial environment in affected countries (Ganie, 2020). The evolution and the fast spread of the Covid-19 signaled the necessity of rapid adaptations of nations and their populations worldwide.

The information on how the pandemic spread quickly across the globe and the extreme efforts of scientists and health care providers to figure out how to effectively treat and contain Covid-19 is a source of worry for all. According to Shanthakumar, Seetharam and Ramesh (2020), with no cure in sight and with the chances of Covid-19 re-emerging even after the world manages to contain the first outbreak, it becomes critical to understand and analyze its disruptions. Some stressors, unique to the disease, include fear of exposure to infection, subsequent risk to infect family members and loved ones, and concerns about educational progression. Initial studies indicate that a significant number of people will experience stressors such as the fear of contagion, the impact of prolonged quarantine and lockdown, and even the death of friends relatives (Wang et al., 2020; Zhou, 2020; Brooks et al., 2020; Xiao, 2020; Felix et al., 2020; Polizzi, Lynn \& Perry, 2020).

During this unprecedented situation, the measures of physical and social distancing that were imposed created the need for a full understanding of how they impact the quality of life of the individuals affected. Our research aims to understand the effects of the current Covid-19 crisis based on the theoretical framework of psychological resilience and the effect of positive emotions, such as hope, on people in conditions of adversity and danger. Under conditions of a worldwide pandemic crisis, it seems like a difficult task to maintain emotional homeostasis and resilience in the face of changeable emotions that make everyday life challenging. However, the drama that everyone affected is called to deal with may become an opportunity for growth and maturity. At the same time, emotional management depends, according to Cyrilnik (2011), on the pillars of resilience in the social network and in the community of each individual.

In this study, we aimed to identify key behaviors and other factors that may contribute to psychological resilience during the pandemic period. Here, we focused on resilience as the self-perceived trait-like ability to cope in the face of adversity, as measured by the Connor-Davidson Risk Scale (CD-RISC) (Connor \& Davidson, 2003). For the purpose of our study, we postulated the following hypotheses:

1. Gender, place of residence, family situation, and level of education all impact, to a greater of a lesser extent, the level of psychological resilience that people exhibit in times of the adversity created through the Covid 19 pandemic.

2. Demographic characteristics such as age, gender, place of residence, and level of education impact to a lesser or greater extent the level of hope that people feel in times of adversity.

3. Trust in state policies and the media increases in times of adversity, leading citizens to comply with extreme measures in order to safeguard the health of their community.

4. Hope is an important predictor of psychological resilience during the Covid19 pandemic crisis. People with high levels of hope exhibit higher levels of resilience and a readiness to adapt to extraordinary circumstances.

\subsection{Literature review}

\subsubsection{Psychological resilience}

According to the American Psychological Association (2020), psychological resilience is defined as the process by which a person gradually adapts to life-threatening conditions, traumatic events, sources of stress and adversity, while a complementary definition stresses the ability to bounce back from adversity and transit from the unpleasant experience back to everyday life (APA, 2020). In the context of determining resilience, the APA refers to the stable personality traits of the individual and its flexibility to adapt, which is related to the human ability to demonstrate mental resilience, regardless of socio-economic and cultural context and personal experiences. The terms "resilience" and "resiliency" 
are differentiated, thus offering different interpretations of their context. On the one hand, "resilience" is based more on environmental influences and is used to describe the positive adaptation of the individual under conditions of adversity. On the other hand, the term "resiliency" identifies all those personality traits of the individual and refers to resilience processes targeted to aid in the design of appropriate interventions (Luthar, 2000). Other definitions present resilience as the ability of a dynamic system to withstand or even to recover from significant threats back to stability, viability, and growth (Masten, 2011). Researchers of resilience agree that the resilience process is influenced by a number of factors, including the traits of the individual, her family, and the wider community (Herrman et al., 2011).

Each and every person has a certain degree of resilience, which is directly related to the protective factors she/he is exposed to. Such factors may be special abilities and skill sets that are found both inside the individual (individual protective resources) but also in the communities (external protective factors) that offset the risk factors. Thus, resilience is considered as an end product of the processes that shield the individual from risks and stressors and allow the person or an entire community to deal with such adversities with self-efficacy (Werner, 2000). In general, researchers increasingly consider resilience not as a defined intrinsic individual trait, but as a variable set of processes that can be cultivated and enhanced. At the same time, they emphasize the interactive processes between the individual and the environment and between risk and protective factors, as the foundation for the development of psychological resilience (Naglieri \& LeBuffe, 2005).

\subsubsection{Resilience in times of the Covid-19 pandemic}

The emergence and quick spread of the Covid-19 virus have disrupted practically every aspect of daily living, causing great insecurity and leaving devastating marks on the psyche of people. The continuous loss of life, the restrictive physical and social distancing measures, the confusion in state protection mechanisms create unprecedented conditions of anxiety, ignorance, doubt, and uncertainty to which individuals are called to adapt in order to keep well or even to survive. At the same time, the Covid-19 crisis touches not only the health but many more aspects of the human existence: Work stability and secure relationships within the family are threatened, as there are risks of abusive relationships within the home due to restrictive measures and negative emotions. The resulting measures of social isolation and the economic uncertainty created through the lockdown measures have led, according to Killgore (2020), to significant increases in mental health concerns, including loneliness, anxiety, depression, and suicidal ideation. Protective factors such as certainty, security, personal values are suspended, while a mechanistic, passive flow of information prevails that has people disoriented and terrorized. In any instance of crisis, pain is common to all people involved, but the way each individual experiences and responds to challenges and difficulties is unique (Cicchetti, 2010).

A crisis is the culmination of a life-threatening situation, a sudden change with a climax of all the negative phenomena that surround it/are involved in it. The return to "normality" or to previous conditions of daily life depends on the reduction or even the complete elimination of the negative changes. Internal concerns are raised for the redefinition of the individual and his new position as an entity in society and community life in relation to his/her internal equilibrium. Thus, the term crisis means an acute situation, which includes unexpected and rapid changes and in which the control is limited, while people exposed to this change are called to modify their behavior and habits in a short period of time (Cicchetti, 2010). According to Brooks (2006), growth is possible even under adversity. In this case, it becomes of importance to recognize one's positive qualities that have not been lost, as well as the new forces that are activated by new experiences. The way that individuals experience a crisis is what creates a potential trauma and not necessarily the crisis itself. Consequently, when the person manages to escape the vicious circle of victimization and takes responsibility for itself, creates a network of communication, filters information, and trusts its individual values and personal path, then it experiences less frustration. The individual will then trust the protective mechanisms provided by the community and will discover pathways to hope and to transcendence (Albert et al., 2020).

The novel coronavirus disease is posing an enormous challenge to the health and emotional well-being of persons across the globe. Research findings indicate that in times of crisis, the effects on mental health are detrimental, although it is not the actual crisis that is the cause of mental disorders, depression, or even suicide. In times of a crisis, many individual elements interact in such ways to form a vulnerable mental state. Recent findings from the OECD (Organization for Economic Cooperation and Development) (2020) survey confirm significant psychological impacts of social distancing and quarantine measures, especially on young people, causing stress, anxiety, and loneliness. This 
is also confirmed by the findings of studies conducted in the UK (Etheridge \& Spanting, 2020) and in the US (McGinty et al., 2020) showing that young adults (aged 18-29) experience a higher level of distress compared to other age groups since the onset of the pandemic.

In recent years, the emphasis in psychotherapeutic practices is placed more on the healthy elements of the self and positive emotions rather than on the pathology and the symptoms (Galanakis, 2011). In this respect, the individual is given the opportunity to highlight his/her strong and dynamic elements, claim responsibility for him-herself and his feelings, and redefine his/her relationships in terms of security and respect (Fredrickson, 2009). According to Raghavan et al. (2020), psychological resilience is not only based on the traits and abilities of the individual to handle crises but also on the interdependence of the person with his environment, i.e., the relationships he develops and maintains with important others in his life. Theory, practice, and research seem to conclude that the response to any stressful event will be significantly affected by the person's assessment of the situation, his ability to predict an effective way to address the challenges that the situation poses at the moment, as well as her ability to eventually integrate the experience in a positive way (Everly \& Lating, 2013).

According to Fredrickson (2003), positive emotions such as love, hope, joy, gratitude, fuel psychological resilience. Research indicates that people who show psychological resilience experience positive emotions more often, which broaden their way of thinking in ways that enable them to deal with adverse situations more effectively. Gupton and Slick (1996) believe that persistence and determination, as well as optimism, contribute to resilience (Christman \& Mclellan, 2007). Hope, according to Snyder et al. (1996), can be defined as a cognitive construct that involves goaldirected thinking, which integrates "pathway" and "agenc" components to it (Snyder et al., 1996). Regarding the two components of hope, they were usually presented to be independent constructs (Snyder et al., 2002). On the one hand, the pathways component referred to the ability to use ways to achieve one's goals, while on the other hand, the agency component referred to the belief than one can actually reach his/her goals. In terms of resilience, hope was seen to play the role of a protective factor (Lloyd \& Hastings, 2009; Mednick et al., 2007) in most studies. In the present study, hope was treated as a possible determinant of resilience and adaptation skills.

\section{Method}

\subsection{Sample}

The study took place online in the Spring of 2020, during the lockdown and the social distancing, quarantine measures that were imposed by the state in both Cyprus and Greece due to the outbreak of the Covid-19 pandemic. The participants were 205 men and women, 18 years and older, who responded to an online invitation to answer our survey. The hyperlink of the survey was shared through Social Media platforms (mainly Facebook and Messenger) and by email invitations, in the period April 1st-20th, 2020. Data analysis showed that the majority of our respondents (78\%) were women, and only $22 \%$ were men. Regarding age, place of residence, level of education, and marital status, our findings showed that most participants resided in urban areas (76\%), and half of them (52\%) were single. As far as our respondents' age groups were concerned, almost half of our subjects belonged to the age group 27-39 years old (45\%). The second-largest age group (37\%) consisted of subjects 40-65 years old, whereas younger people (18-26 years) compiled $18 \%$ of our sample. Regarding our sample's level of education, data analysis indicated that the majority (66\%) had a university degree.

\subsection{Procedure-data collection}

All instruments were integrated into one questionnaire that was created on the Anketa platform (https://www.1ka. si/) and consisted of a total of 68 items. A short, introductory note informed the participants about the purposes of our research before they were asked to complete the survey. The introductory note also included brief instructions regarding the Likert-scales. Subjects completed the Personal Information Form (PIF), the CD-RISK and the AHS Scale, and the CAQ (Covid19-Adaptation-Questionnaire), which was created for the purposes of this study. 


\subsubsection{Instruments}

A five-item Personal Information Form (PIF) was used to collect demographic data regarding the participants' age, gender, marital status, place of residence, and educational level. This was followed by the first questionnaire, namely, the Conner-Davidson Resilience Scale (CD-Risk), which assesses levels of psychological resiliency as an indicator of peoples' response to stressful situations; the second instrument was Snyder's Adult-Hope-Scale (AHS). Our third and last instrument was a self-report questionnaire constructed by the researchers solely for the purpose of the study in order to assess the subjects' perception of their ability to adapt to sudden changes, the perceived changes in their daily life due to the quarantine, and their level of trust to state policies and the media regarding Covid-19. We applied all the instruments in their Greek versions.

The Conner-Davidson Resilience Scale (CD-Risk) was created by Davidson in 2003 and aimed to explore psychological resilience. It comprises of 25 items, each rated on a 5-point Likert scale (0-4) with higher scores reflecting greater resilience (Connor \& Davidson, 2003). The highest score possible on the CD-Risk scale is 100, and the lowest is 1. Items are grouped into the following five factors. The first factor ( 8 items) reflects the notion of personal competence, high standards, and tenacity. The second factor (7 items) is related to trust in one's intuition, tolerance of negative affect, and the strengthening effects of stress. The third factor ( 5 items) reflects positive acceptance of change and secure relationships. The fourth factor (3 items) reflects control. The fifth factor ( 2 items) reflects spiritual influences. The scale was validated using different samples (five in clinical settings and one in the community) (Gonzales et al., 2015). The authors reported that the scale has high internal consistency, good test-retest reliability, and adequate convergent and discriminant validity (Cronbach's $\alpha=0.89$ ). Although the CD-RISK is considered one of the best-known instruments in the field of resilience assessment, Campbell-Stills and Stein (2007) removed items with low or inconsistent loadings or those with overlapping contents and concluded that the structure of the scale is unidimensional, retaining only 10 of the original 25. In our research, we applied the original version in order to gain a better understanding of the resiliency indicators.

Our second instrument was the Adult-Hope-Scale (AHS) (Snyder, 1996), which is a 12-item measure that assesses the respondent's level of hope. The Adult-Hope-Scale scale is divided into two subscales that comprise Snyder's cognitive model of hope: (1) Agency (i.e., goal-directed energy) and (2) Pathways (i.e., planning to accomplish goals). Of the 12 items, 4 make up the Agency subscale, and 4 make up the Pathways subscale. The remaining four items are fillers. Each item is answered using an 8-point Likert-type scale ranging from Definitely False to Definitely True. The scoring of the scale is as follows: Items 2, 9, 10, and 12 comprise the subscale of the Agency, whereas items 1, 4, 6 and 8 constitute the subscale of the Pathways or the design. Researchers may either look at the results at the subscale level or combine the two subcategories to create an overall score of hope. Total hope scale scores range from a minimum of 8 to a maximum of 64 . For the subscales, agency, and pathways, scores range from a minimum of 4 to a maximum of 32, whereas high scores reflect high levels of hope (Snyder et al., 1991). Snyder and his associates (1996) tested the reliability of this scale in four studies in a sample that included 444 American students. The authors found Cronbach alphas of 0.79 to 0.95 for the overall state hope scale, 0.79 to 0.95 for the agency subscale, and 0.59 to 0.93 for the pathway subscale, therefor demonstrating strong support for the internal reliability (Lopez et al., 2000). Let it be noted that the authors recommend that when administering the scale, it is called "The Future Scale".

Our third instrument was a questionnaire created by the researchers for the purpose of this study. We named it the Covid19-Adaptation-Questionnaire (CAQ). It comprises 18 items, each rated on a seven-point Likert scale. Items aim to explore the ability to adapt under the conditions and the measures of the specific crisis, namely the Covid-19 pandemic. Amongst other things, items addressed citizens' internal resources to overcome the crisis on a personal level and their flexibility to rebound, the citizens' trust of governmental measures to address the crisis, social trust, and the feeling of state protection during the crisis. The 18 items were grouped in three main categories, namely items 1-8 "Adaptation to sudden change", items 9-13, "Changes in daily life due to the quarantine", and items 13-18 "Trust in state policies and the media". Subjects responded on a 7-point Likert scale ranging from "totally disagree" to "totally agree". The highest possible score on the CAQ is 96.

\subsubsection{Data analysis}

We analyzed our data with the use of the Statistical Package for Social Sciences (SPSS) version 25.0. We 
summarized and analyzed our data through descriptive statistics, such as mean, standard deviation, frequencies, and percentages, to describe the participants' demographic characteristics. Distributions of frequencies and percentages were calculated in order to analyze data obtained from the participants' personal information form. The level of significance ( $\mathrm{p}$-value) in all three questionnaires was set at $\mathrm{p}<0.05$.

In order to examine our four hypotheses, we implemented the following data analysis methods: The t-test, the two-way ANOVA, and the multivariate ANOVA (MANOVA) to compare the means between subjects' demographic characteristics and their impact on their overall resilience, hope, and adaptation scores. Our participants' gender, age, family status, place of residence, and level of education were treated as independent variables to examine their impact on overall psychological resilience and hope.

In order to test our fourth hypothesis and to establish whether hope can impact and predict psychological resilience, we applied the Pearson correlation coefficient in order to explore whether correlations (positive or negative) between psychological resilience, the positive feeling of hope, and the ability to adapt under adversity were statistically significant.

Table 1. Descriptive indicators and the internal consistency values of the tools

\begin{tabular}{ccc}
\hline Questionnaires & Authors & Our research \\
\hline & Cronbach alpha & Cronbach alpha \\
CD-risk-resilience scale & 0.89 & 0.92 \\
AHS - adult hope scale & $0.79-0.95$ & 0.94 \\
CAQ-Covid19-adaptation-questionaire (overall) & 0.86 \\
Category 1: Adaptability to change & 0.83 \\
Category 2: Changes in daily life & 0.73 \\
Category 3: Trust in state policies & & 0.77 \\
\hline
\end{tabular}

Table 1 shows the internal consistency of the three questionnaires; that is, how closely related a set of items are as a group. The general rule of thumb is that a Cronbach's alpha of 0.70 and above is good, 0.80 and above is better, and 0.90 and above is best. Compared to the Cronbach alpha of the original questionnaires, the value of Cronbach alpha in our Greek versions for the CD-Risk was slightly higher (0.92) and above 0.90, which is considered very good. The same also stands for the internal consistency of the AHS-Greek version with a value of 0.94 . The internal consistency of our specially designed CAQ (Covid19-Adaptation-Questionnaire) had a value of 0.86, which indicates good scale reliability. All three categories of items that we created also had an acceptable Cronbach's alpha value, namely Adaptability to change 0.83 , Changes in daily life 0.73 , and Trust in state policies 0.77 .

\section{Results}

\subsection{Resilience}

In examining our first hypothesis, namely that demographic characteristics such as gender, age, family situation, place of residence, and level of education all impact, to a greater of a lesser extent, the level of psychological resilience that people exhibit in times of adversity (in this case created through the Covid-19 pandemic), our data analysis showed that the overall resilience score of our sample tended to be relatively high ranging between 87 and 93/100 points. The gender of our sample could not predict their level of resilience as we detected no significant differences between our participating men (Mean $=89)$ and women $($ Mean $=90, \mathrm{SD}= \pm 2.87)$. The same stands also for their place of residence and their level of education, where no significant differences were detected in relation to the sample's level of overall psychological resilience. 
Table 2. Overall resilience scores, age and family situation

\begin{tabular}{cccccc}
\hline Age Groups & Resilience (Mean) & SD & Family Situation & Resilience (Mean) & SD \\
\hline $18-26$ & 87.8 & \pm 3.45 & Single & 88.3 & \pm 2.44 \\
$27-39$ & 88.4 & \pm 2.88 & Married & 90.1 & \pm 1.90 \\
$40-55$ & 92.7 & \pm 1.29 & Widowed & 96 & \pm 1.78 \\
$56-64$ & 93.8 & \pm 1.33 & Divorced & 98.1 & \pm 0.88 \\
\hline
\end{tabular}

However, as shown in Table 2, data analysis indicated that the age and family situation of our participants were significant determinants of resilience in times of crisis. Compared with the younger age group, 18-39 years of age (Mean $=88.0, \mathrm{SD}= \pm 3.45$ for 18-26 years old and $\mathrm{SD}= \pm 2.88$ for 27-39 years of age respectively), our participants older than 40 years had significantly higher scores on the CD-Risk (Mean $=93.2, \mathrm{SD}= \pm 1.78$ ), thus demonstrating that resilience increases significantly as age progresses $(p<0.05)$. Furthermore, in regard to their family situation, our analysis showed that widowed and divorced subjects had significantly higher levels of overall resilience than those who were single or married. Indeed, married subjects exhibited higher resilience scores (Mean $=90.1, \mathrm{SD}= \pm 1.90$ ) than single participants (Mean $=88.3, \mathrm{SD}= \pm 2.44)$, but these differences were not significant $(\mathrm{p}>0.05)$. The fact that widowed $(\mathrm{Mean}=96$, $\mathrm{SD}= \pm 1.78$ ) and divorced (Mean $=98.1, \mathrm{SD}= \pm 0.88$ ) participants exhibited higher scores of resilience than married or single subjects was found to be statistically significant $(\mathrm{p}<0.05)$.

\subsection{Hope}

The results of our sample regarding "hope" derived from the application of Snyder's Adult Hope Scale (AHS). Our sample's mean score indicated that the participants' overall level of hope ranged from 53 as the lowest and 62 as the highest score. The mean of our sample on the AHS was $55.5(\mathrm{SD}= \pm 7.2)$, thus showing that our sample's overall level of hope at the time of our study was in the range from medium to high. In examining our second hypothesis, namely that demographic characteristics such as age, gender, place of residence, and level of education impact to a lesser or greater extent the level of hope that people feel in times of adversity, we were not able to establish any indications that gender, age or place of residence have a significant impact on the positive feeling of hope.

Table 3. Overall hope scores, level of education and family situation

\begin{tabular}{cccccc}
\hline Level of education & Hope (Mean) & SD & Family situation & Hope (Mean) & SD \\
\hline High School & 53.2 & \pm 7.50 & Single & 54.1 & \pm 7.11 \\
College Degree & 54.1 & \pm 7.02 & Married & 55.8 & \pm 7.37 \\
Master's Degree & 58.0 & \pm 6.44 & Widowed & 57.0 & \pm 1.41 \\
Ph.D. & 58.1 & \pm 7.21 & Divorced & 60.5 & \pm 4.98 \\
\hline
\end{tabular}

Nevertheless, as shown in Table 3, the educational level seems to be a significant predictor for the positive feeling of hope. Participants with a higher level of education such as a Master's (Mean $=58, \mathrm{SD}= \pm 6.44$ ) or a Ph.D. degree $($ Mean $=58.1, \mathrm{SD}= \pm 7.21)$ were significantly more hopeful than subjects with only a high school education $(\mathrm{Mean}=$ $53.2, \mathrm{SD}= \pm 7.50)$ or a college degree $($ Mean $=54.1, \mathrm{SD}= \pm 7.02)(\mathrm{p}<0.05)$. Furthermore, and as shown above, the highest hope scores were exhibited by participants who had gone through a divorce (Mean $=60.5, \mathrm{SD}= \pm 4.98)$. Indeed, their scores on the AHS were significantly higher than those subjects that were single (Mean $=54.1, \mathrm{SD}= \pm 7.11$ ) or married $($ Mean $=55.8, \mathrm{SD}= \pm 7.37)(\mathrm{p}<0.05)$. 


\subsection{Adaptation and trust in state policies in times of quarantine}

In order to analyze the data collected through our third instrument, the CAQ, on the three categories (Adaptation to change, Changes in daily life, and Trust in state policies and media), results from the Likert-scales were grouped into three main answer categories, namely "agree" (by adding the percentages of those who answered either "totally agree", or "agree" or "agree with a little") and "disagree" (by adding the percentages of those who answered "totally disagree", or "disagree" or "disagree a little").

Table 4. Category 1: Adaptation to change

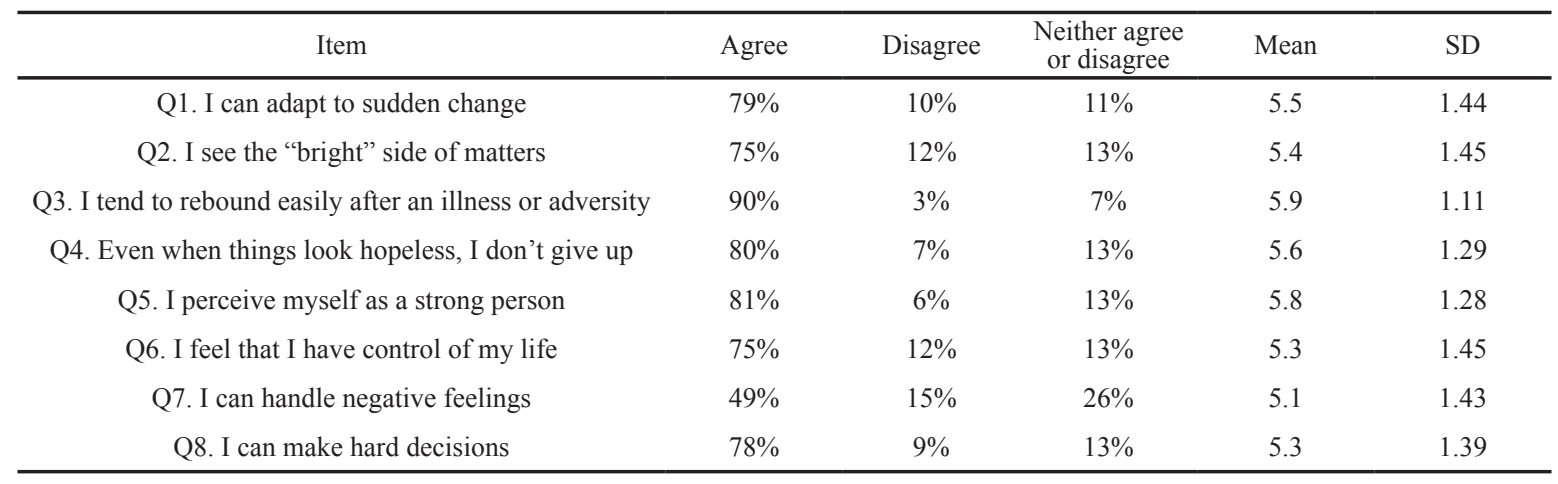

As we can see from our data, a large majority of our participants $(90 \%)$ perceived themselves as individuals who tend to rebound easily after adversity $($ Mean $=5.5, \mathrm{SD}=1.44)$, and $79 \%$ considered themselves as easily adaptable to changes (Mean $=5.4, \mathrm{SD}=1.45)$. At the same time, they described themselves as hopeful and persistent $(80 \%)(\mathrm{Mean}$ $=5.6, \mathrm{SD}=1.29)$, strong $(81 \%)($ Mean $=5.8, \mathrm{SD}=1.28)$, in control of their lives $(75 \%)(\mathrm{Mean}=5.3, \mathrm{SD}=1.45)$ and as decision-makers $(78 \%)($ Mean $=5.3, \mathrm{SD}=1.39)$. As shown in the table above and in comparison with the other seven items of the category, our participants had lower confidence in handling negative feelings $(49 \%)(\mathrm{Mean}=5.1, \mathrm{SD}=$ 1.43). There is a statistically significant difference in the ability to adapt during the crisis between younger and older participants $\left[\mathrm{F}_{(4,204)}=3.2, \mathrm{p}<0.001\right]$. Older subjects $(40-65$ years old) (Mean $=91.7, \mathrm{SD}=12.3)$ demonstrated a higher level of adaptability than the younger $($ Mean $=84.5, \mathrm{SD}=14.6)$.

Table 5. Category 2: Changes in daily life

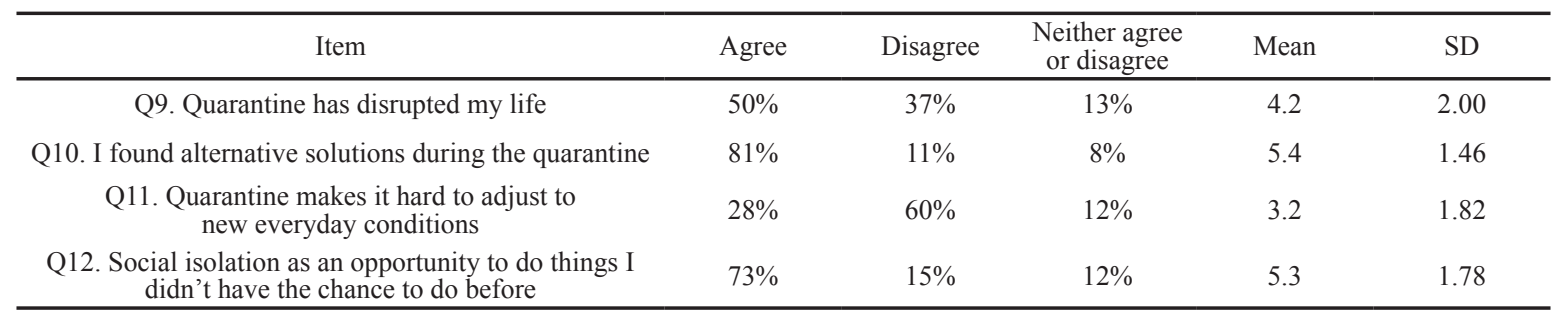

Regarding our second category, "Changes in daily life", as shown in Table 5, half of our participants (50\%) (Mean $=4.2, \mathrm{SD}=2.0$ ) perceived the strict social isolation measure of the quarantine as a disruption to their life, but also more than one third (37\%) did not perceive the measure as such and did not find it hard to adjust to such extreme circumstances $(60 \%)($ Mean $=3.2, \mathrm{SD}=1.82)$. As a matter of fact, the responses of our sample showed that the majority of our subjects $(81 \%)($ Mean $=5.4, \mathrm{SD}=1.46)$ were able to find alternative solutions during the quarantine, while $73 \%$ of the respondents felt that social isolation was an opportunity to do things that they did not have a chance to integrate 
into their daily routine under circumstances of normalcy (Mean $=5.3, \mathrm{SD}=1.78$ ). We established no significant differences in regards to the level of education or the place of residence. However, family status was a significant determinant of our subjects' overall ability to adapt in the face of adversity $\left[\mathrm{F}_{(3,201)}=4.318 \mathrm{p}<0.006\right]$. Married participants of both genders (Mean $=94.7, \mathrm{SD}=11.48$ ) displayed higher levels of adaptation to the quarantine than those who were single (Mean $=84.11, \mathrm{SD}=15.08)$, divorced $($ Mean $=89.9, \mathrm{SD}=13.29)$ or widowed (Mean $=84.0, \mathrm{SD}$ $=11.31)$. As in Category 1, we detected a significant difference between the age groups $\left[\mathrm{F}_{(4,204)}=2.042 \mathrm{p}<0.05\right]$. Older participants (age $40+$ ) perceived the time of the quarantine as less disruptive to their routine (Mean $=25.9, \mathrm{SD}=4.84$ ) than our younger age group $(18-39)($ Mean $=23.37, \mathrm{SD}=12.6)$.

Table 6. Category 3: Trust in state policies and the media

\begin{tabular}{|c|c|c|c|c|c|}
\hline Item & Agree & Disagree & $\begin{array}{c}\text { Neither agree } \\
\text { or disagree }\end{array}$ & Mean & SD \\
\hline $\begin{array}{l}\text { Q13. If I follow quarantine policies, it will be beneficial } \\
\text { for me in the long-run }\end{array}$ & $62 \%$ & $15 \%$ & $23 \%$ & 5.1 & 1.72 \\
\hline $\begin{array}{l}\text { Q14. The state is doing everything possible to address } \\
\text { the pandemic }\end{array}$ & $50 \%$ & $39 \%$ & $11 \%$ & 4.2 & 2.08 \\
\hline Q15.I feel that there is no protection by the State & $40 \%$ & $35 \%$ & $25 \%$ & 4.2 & 1.85 \\
\hline Q16. Social trust to the state policies is shaken & $45 \%$ & $32 \%$ & $23 \%$ & 4.3 & 1.90 \\
\hline $\begin{array}{l}\text { Q17. Mass Media have a positive effect on my attitude } \\
\text { towards the pandemic }\end{array}$ & $21 \%$ & $65 \%$ & $14 \%$ & 5.1 & 1.72 \\
\hline
\end{tabular}

As far as our third category is concerned, our data analysis showed that our samples' views regarding state policies were indeed divided. More than half of our participants (62\%) felt that in the long-run, the quarantine would protect them against Covid-19. Interestingly enough, though, almost one-quarter of our subjects (23\%) had no opinion on this matter (Mean $=5.1, \mathrm{SD}=1.72$ ). And even though people followed the measures, $40 \%$ of our participants did not feel securely protected by the state during that time, whereas we observed the same trend as with the previous item above, namely that $25 \%$ of the subjects had no opinion on the matter (Mean $=4.2, \mathrm{SD}=1.85$ ). Half of our participants $(50 \%)$ felt that the state was doing everything in its power to protect its citizens during the Covid-19 crisis, but almost as many participants $(40 \%)$ felt the exact opposite (Mean $=4.2, \mathrm{SD}=2.08$ ). Indeed, $45 \%$ of our participants claimed that their social trust to state policies was shaken, and only one-third of our participants (32\%) still felt trust toward the state's extraordinary measures. We detected no statistically significant differences between the genders, or as far as the place of residence and level of education was concerned. However, family status seemed to be a determinant of trust in state policies $\left[\mathrm{F}_{(3,201)}=4.260 \mathrm{p}<0.006\right]$. Married subjects (Mean $=20.83, \mathrm{SD}=5.54$ ) showed significantly more trust in state policies during the quarantine than our single (Mean $=18.30, \mathrm{SD}=5.38)$, divorced $($ Mean $=18.66, \mathrm{SD}=5.61)$ or widowed (M.O.13,50, $\mathrm{SD}=0.70)$ subjects.

When it came to the role that the Mass Media have played during the peak of the Covid-19 crisis, it was quite clear that the majority of our subjects $(65 \%)$ perceived their role as detrimental in regards to shaping their attitude toward the pandemic $($ Mean $=5.1, \mathrm{SD}=1.72)$.

\subsection{Resilience, hope and adaptation during the Covid-19 crisis}

Our third hypothesis postulated that the positive feeling of hope could be a determinant of psychological resilience and adaptation during times of adversity. We applied a correlation analysis between the variables "Psychological Resilience", "Hop" (AHS), and "Adaptation" by comparing our sample's means on the questionnaires CD-Risk, AHS, and the CAQ. 
Table 7. Resilience, hope and adaptation

\begin{tabular}{ccccc}
\hline & & Resilience & Hope & Adaptation Covid-19 \\
\hline Resilience & Pearson Correlation & 1 & $0.707^{* *}$ & $0.725^{* *}$ \\
& Sig. (2-tailed) & & 0.000 & 0.000 \\
& $\mathrm{~N}$ & 205 & 205 & 205 \\
Hope & Pearson Correlation & $0.707^{* *}$ & 1 & $0.546^{* *}$ \\
& Sig. (2-tailed) & 0.000 & & 0.000 \\
& $\mathrm{~N}$ & 205 & 205 & 205 \\
Adaptation & Pearson Correlation & $0.725^{* *}$ & $0.546^{* *}$ & 1 \\
& Sig. (2-tailed) & 0.000 & 0.000 & 205 \\
\hline
\end{tabular}

**. Correlation is significant at the 0.01 level (2-tailed).

As shown in Table 7, our findings indicated a significant positive correlation between our subject's means on the Adult Hope Scale, their mean scores on the Resilience Scale as well as their mean score on the Covid19-AdaptationQuestionnaire $(r=0.707, p<0.01)$. A significant positive correlation was also detected between Adaptation during the Covid-19 quarantine measures and hope $(r=0.546, p<0.01)$. At the same time, participants with higher levels of Resilience showed a significantly higher ability to adapt during the time of the Covid-19 adversity $(r=0.858, p<0.01)$.

In effect, when participants exhibited higher scores on Hope, then their Resilience also increased. The reverse was also proven significant, i.e., subjects with higher scores on Resilience exhibited at the same time elevated scores on Hope but also a significantly higher ability to adjust to the extreme measure of the state-imposed quarantine.

\section{Discussion}

The aim of the present study was to explore psychological resilience and adaptation in relation to the positive feeling of hope under the circumstances of quarantine during the Covid-19 pandemic crisis. We postulated four hypotheses, and through data analysis, we were able to demonstrate significant associations between psychological resilience and hope as well as between psychological resilience, hope, and adaptation under circumstances of adversity in a representative sample of 205 Cypriot and Greek adults (over 18 years of age). The majority of our participants demonstrated medium to high scores on the Resilience Scale (CD-Risk), and data analysis confirmed a part of our first hypothesis, namely that demographic characteristics such as age and family status play a significant role in our sample's overall levels of psychological resilience. According to our findings, compared with our younger age group, our participants older than 40 years demonstrated significantly higher scores on the CD-Risk, thus showing that in our research, the Covid-19 crisis seems to have affected particularly younger age groups, since these subjects had lower scores on the resilience scale than older participants. The difference between the age groups was statistically significant, thus, indicating that resilience to the crisis increases as the mean age of the sample progresses. This is also confirmed by the findings of studies conducted in the UK (Etheridge \& Spanting, 2020) and in the US (McGinty et al., 2020) showing that young adults (aged 18-29) have been experiencing a higher level of distress compared to other age groups since the onset of the pandemic. Furthermore, widowed and divorced subjects exhibited higher resilience during the adversity of the lockdown and the quarantine than those who were single or married. This last finding comes in a surprise since most studies on resilience emphasize the positive role of the family, social, and community network in cultivating and maintaining resilience. However, a possible interpretation could be found in Coutu's (2002) understanding of resilient individuals, who postulated that resilient individuals have more confidence than those less resilient in their ability to persevere. They have done so before, and therefore, they anticipate rather than fear change and challenges. Older people, as well as those who have gone through the process of separation through death or divorce, experience the same difficulties and stressors as everyone else; they are not immune or hardened to stress, but they have learned through their experiences to deal with life's inevitable difficulties to adapt and to re-establish the equilibrium, and this ability is 
what sets them apart. According to Fontes and Neri (2015), the field of lifespan development has adopted the concepts of plasticity and capacity for cognitive reserve, considering resilience as the maintenance of normal development, despite the risks, and recovery from traumatic situations (Baltes, 1997; Staudinger et al., 1993). Ong (2009) detects two underlying conditions of the concept of resilience as patterns of adaptation under conditions of risk or adversity: exposure to significant risk and evidence of positive adaptation to threats to development. In this context, positive adaptation refers to successful development despite the risks, namely the ability to deal with stress, including the capacity to minimize the impact of the stressful event; the capacity for a rapid rebound from trauma; and, in the long term, the ability to minimize negative responses and $\mathrm{c}$ to promote positive consequences and behaviors that enable the individual to overcome adversity. Relevant research on resilience has shown high resilience in the elderly and middleaged (Nygren et al., 2005). The results showed that higher levels of resilience were associated with increasing age, suggesting that younger people are not always as resilient as those who are older. Gooding's findings also revealed that older individuals with high levels of psychological resilience show increased levels of longevity (Gooding et al., 2012). Thus, older people are considered psychologically resilient when they do not succumb to adversity, but rather exhibit the capacity for successful adaptation, i.e., when they can manage successfully stressful events or recuperation to previous or baseline levels of objective or subjective well-being after exposure to an adverse event. Indeed, regarding the ability to adapt while experiencing the quarantine, our findings demonstrate that our subjects belonging to older age groups exhibited higher adaptability than our younger participants. Older participants (age 40+) perceived the time of the quarantine as less disruptive to their routine than subjects aged below 40 years. In the face of adversity, as Kobasa (1982) pointed out, as early as 1982, our findings showed that older generations are able to change the meaning of adversity, reduce the level of (perceived) danger caused by stressful events, reduce not only their exposure but also their negative reactions to such events, maintain positive self-esteem and self-efficacy and create opportunities to revert the effects of stress. These actions contradict vulnerability, are understood as the lack of psychological coping resources, and manage the undesirable effects of the risks and possible threats to adaptation. In this sense, they enable people of older generations who possess such resources to overcome biological, socio-economic, and psychological risk factors.

Recently, the importance of studying resilience or resilient groups has been emphasized by proponents of positive psychology. Positive psychology focuses more on personal positive thoughts and experiences. Studies devoted to these topics include a focus on the experiences of delight and happiness, and on variables such as optimism and hope. In this study, we addressed hope and its relationship to psychological resilience. Snyder (1994) argued that a person who has a high level of hope is more likely to accept challenges and places his/her focus on success rather than on failure, as well as and on the possibility to reach his or her goal, and maintains a positive emotional stance. On the other hand, a person who has a low level of hope does not do his or her best, focuses on failure and on the impossibility of goal attainment, and keeps a negative emotional stance. Consequently, these characteristics of hope could play an important role in overcoming adverse circumstances. We assessed our sample's level of hope utilizing the Adult-Hope-Scale, whereas our findings showed that our sample's overall level of hope at the time of our study was medium. Further data analysis for examining our second hypothesis has not been able to establish that demographic characteristics such as age, gender, place of residence, and family status were determinants of our sample's levels of hope. However, our participants' level of education was found to be a significant predictor for their positive feeling of hope. According to Folkman (2010), the relationship between hope, sense of coherence, and coping is dynamic and reciprocal; each in turn supports and is supported by the others. Furthermore, individuals adjusted their hope levels based on their relative level of success or failure (Feldman et al., 2009). In a comparison between high and low hope students, it was found that high hope students succeeded in their studies more than their classmates (Snyder et al., 1997) and revealed effective problem-solving styles (Chang, 1998). In this respect, it is perhaps self-efficacy and self-awareness deriving from having achieved a higher education that, in accordance also with Celik's (2015) findings, both seemed to predict both hope components, namely pathways and agencies. Furthermore, self-esteem as a personal characteristic of individuals who survived in the face of adverse or risky situations was conceptualized by Mruk (1999) in the understanding that the way people feel about themselves (self-esteem level) was both dependent on seeing themselves as worthwhile and accepted by others and also their judgments about competence in several domains. As for the relationship between resilience and self-esteem, selfesteem could be listed both as a protective factor and an outcome of resilience. As an example, individuals having high self-esteem were reported to be more resilient in the face of challenges (Hames \& Joiner, 2012).

In our third hypothesis, we postulated that trust in state policies and the media increases in times of adversity, 
leading citizens to adapt to extreme measures in order to safeguard the health of their family and their community. The overall results of our sample acquired through the implementation of the Covid19-Adaptation-Questionnaire indicated that a large majority of our participants perceived themselves as individuals who tended to rebound easily after adversity and were easily adaptable to changes. Even though half of our respondents perceived the strict social isolation measure of quarantine as a disruption to their life, the majority also claimed that they had no difficulty in making the necessary adjustments to the new reality. One critical aspect of resilience that has been emphasized in literature at the individual level is its improvisational nature, the willingness to pursue alternative action experimentally (Barrett, 2004), to regain balance and to keep going despite adversity, and to find meaning amidst confusion and tumult. Whether resilience has to be considered an adaptive ability in the face of actual stressing experiences or rather a personal characteristic describable within a personality profile is, according to Gianesini (2011), still unclear. The results of our sample indicated that a large majority of our participants adapted quickly to the social isolation measures that were imposed through state policies. Indeed, their responses showed that they felt that during the quarantine, they were able to find alternative solutions to smooth out the impact of the measure in their daily routine. A large percentage of our respondents also claimed that social isolation presented them with the opportunity to do things that they did not have the chance to do before the implementation of the social distancing and isolation measures. More than half of our participants felt that keeping in line with these measures would protect them, in the long run, from contracting the Covid-19 virus. Our findings from the Covid19-Adaptation-Questionnaire showed consistency with our findings on the CD-Risk Resilience scale, and regarding as far as the age groups were concerned, i.e., our data analysis indicated that participants over the age of 40 demonstrated a significantly higher level of adaptation to the conditions of social and physical isolation than younger subjects. At this point, one could pose the question whether the subjects' readiness to adapt was due solely to fear of contagion (since they belonged to older age groups), or whether it was due to their trust in the state policies and the information by the mass media, that propagated and supported those policies. Indeed, over half of our participants seemed to have followed the quarantine measures due to their belief that, in the long-run, these would protect them against the virus. However, not everyone was convinced; Almost half of our participants felt that the state failed to implement the right policies to protect them and that their trust in state policies at that time was challenged. In view of our findings, we cannot claim that our third hypothesis was confirmed, since our sample's trust in state policies and the media did not increase during this time of extreme adversity. Compliance with the measures seems to have been a result of people's need to safeguard not only their own health but also the health and well-being of their family and community. Furthermore, the majority of the participants in our study felt that the role of the media in this crisis was not constructive, as they perceived that the media did not have a positive effect on their attitude and on their behavior during the quarantine. The development of a sense of distrust toward state policies and the mass media during the Covid-19 crisis seems to be a trend not only in Cyprus and Greece but also in other countries. Research carried out by Fletcher et al. (2020) in May of 2020 found that trust in the UK government as the origin of information about coronavirus had declined substantially since April. In fact, $48 \%$ of the participants rated the government relatively trustworthy in late May, down from $67 \%$ six weeks earlier. Trust in news organizations had also declined, from 57\% to $46 \%$. According to the authors, these drops are large and significant (as is the drop for politicians), and much more substantial than the significantly smaller changes around other institutions. On the other hand, survey results obtained in the USA were different: Subjects were asked about their opinion regarding four elements of the news media's coverage of the coronavirus outbreak: (a) whether the news coverage is giving them the information they need, or not; (b) whether it is largely accurate or inaccurate; (c) whether it is benefiting the public or the media themselves, and last but not least (d) whether it is helping or hurting the country. Overall results indicated that more Americans regard the news media as fulfilling the above key roles during this crisis than not, a finding also supported by other recent findings showing that most Americans believe that the news media are doing fairly well in covering the outbreak more generally.

Our research findings confirm our fourth and last hypothesis, indicating a significant positive correlation between our participants' scores on the Adult-Hope-Scale with those on the Resilience Scale. In other words, when subjects exhibited higher scores on Hope, their overall Resilience was also higher. Similarly, higher scores on Resilience resulted in elevated scores on Hope. At the same time, participants with higher levels of resilience showed a significantly higher ability to adapt during the time of the Covid-19 to social and physical distancing measures in the form of governmentmandated social distancing measures, even the quarantine.

The findings of our study have to be seen in light of some possible limitations. This research was carried out 
completely online, since it took place during the Covid-19 lock-down, physical distancing measures. As such, it was subject to all the possible limitations of online surveys, such as, for example survey fraud. Having this in mind, our effort focused on limiting the length of our survey to a total of 43 items in order to avoid confusion and the possibility of fake answers. Another drawback of online surveys is that certain populations are less likely to have internet access, thus restricting their access to online questionnaires. Indeed, since our call for the participation in our research was made primarily through the social media, our sampling ran the risk of having those participants who either had a good connectivity to the internet or who are usually active on social media platforms. Since we had no control in regards to the demographics of our participants, we closed the survey when we had reached a number of 205 participants. This resulted in an overrepresentation of women (78\%). Concerns regarding the imbalance between the number of men and women participants have also been raised in other studies (McCray et al., 2005). In this case, our results may have been influenced by this gender imbalance as far our first two hypotheses were concerned, where we postulated that gender could impact psychological resilience and hope in times of adversity. However, neither in the assessment of resilience nor in the assessment of hope did our data analysis detect any significant differences between men and women of our sample. Last but not least, we did not carry out an IP address verification for our participants, so we did not engage in any processes to check and eliminate cases where multiple repetitive or similar IP addresses were involved. This is a point of consideration for improvements when planning our next research.

\section{Conclusion}

This paper considered the effects of the quarantine during the current Covid-19 crisis based on the theoretical framework of psychological resilience and the effect of positive emotions, such as hope, on people in conditions of adversity and danger. In a nutshell, we could summarize the results of our study as follows: First, the level of our participants' resilience was medium to high during the time of the quarantine, whereas people older than 40 and individuals who had resolved other crises in their lives, such as separation by death or divorce exhibited higher levels of resilience than the younger ones. Second, during the period of the quarantine, the positive feeling of hope was also kept medium to high, with people with higher education displaying a higher level of hope as compared to those with just high school or college education. Third, older people, 40 years and older, more so than the younger ones, were able to adapt with more ease to the adverse daily life conditions during the quarantine. Married participants of both genders displayed higher levels of adaptation to the quarantine than those who were single, divorced, or widowed. Fourth, almost half of our participants felt that the state failed to implement the right policies to protect them and that their trust in state policies at that time was challenged. Fifth, mass media credibility was relatively low, as more than half of the subjects felt that the role of the media in this crisis was not constructive, and the media did not have a positive impact on their attitude(s) and on their behavior during the quarantine. Last but not least, a positive correlation was established between resilience, hope, and adaptability. It appears that the positive feeling of hope is more readily activated when the person has pre-existing experiences of dealing with adversity, through which he/she finds the inner resources and strength not only to cope but also to rebound.

Important knowledge gained from the COVID-19 pandemic is perhaps the call for understanding the vulnerability of individuals, communities, and societies in order to establish reliable, targeted guidance and advance warnings. Various studies highlight the multidimensional nature of resilience, demonstrating a complex interaction between individual and environmental factors. Such models usually involve a combination of risk factors (individual, environmental, and incremental) and protective factors (personal traits, interpersonal relations, and support), and results in successful adaptation, recovery, and psychological well-being (Smith \& Hayslip, 2012; Ryff et al., 2012). Indeed, protective factors, bolstering psychological resilience should be a primary public health emphasis during the Covid-19 pandemic. Additionally, and in view of the fact that the state has failed to convince citizens that the implemented policies would protect them against the pandemic, it would be interesting to research how communities respond to potential future crises and whether the policies that state and society implement encourages or discourages the activation of individual processes of psychological resilience. 


\section{References}

Albert, W., Cheryl, C., \& George, S. Everly Jr. (2020). COVID-19: Peer support and crisis communication strategies to promote institutional resilience. Annals of Internal Medicine, 172(12), 822-823.

American Psychological Association. (2020, February 1st). Building your resilience. https://www.apa.org/topics/ resilience.

Baltes, P. B. (1997). On the incomplete architecture of human ontogeny. Selection, optimization, and compensation as foundation of developmental theory. American Psychologist, 52(4), 366-380

Barrett, F. (2004). Coaching for resilience. Organization Development Journal, 22(1), 93-96.

Brooks, S. K., Webster, R. K., Smith, L. E., Woodland, L., Wessely, S., Greenberg, N., \& Rubin, G. J. (2020). The psychological impact of quarantine and how to reduce it: A rapid review of the evidence. Lancet, 395(10227), 912920.

Campbell Sills L, \& Stein MB. (2007). Psychometric analysis and refinement of the connor-davidson resilience scale (CD-RISC): Validation of a 10-item measure of resilience. Journal of Trauma Stress, 20(6), 1019-1028.

Carpio Carmen. (2020). STP Covid-19 Emergency Response Project. MENA Report. https://documents.worldbank.org/ en/publication/documents-reports/documentdetail/406371585129780224/project-information-document-stp-covid19-emergency-response-project-p173783.

Chang, E. C. (1998). Hope, problem-solving ability, and coping in a college student population: Some implications for theory and practice. Journal of Clinical Psychology, 54(7), 953-962

Connor, K. M., \& Davidson, J. R. (2003). Development of a new resilience scale: The connor-davidson resilience scale (CD-RISC). Depression and anxiety, 18(2), 76-82.

Coutu, D. L. (2002). How resilience works. Harvard Business Review, 80(5), 46-55.

Cyrilnik, B. (2011). Resilience: How Your Inner Strength Can Set You Free from the Past. London: Penguin Publishing Group.

Duysal Askun Celik, Fatih Çetin, \& Erkut Tutkun. (2014). The role of proximal and distal resilience factors and locus of control in understanding hope, self-esteem and academic achievement among Turkish pre-adolescents. Current Psychology, 34(2), 321-345.

Emanuel, E. J., Persad, G., Upshur, R., Thome, B., Parker, M., Glickman, A., Zhang, C., Boyle, C., Smith, M., \& Phillips, J. P. (2020). Fair allocation of scarce medical resources in the time of COVID-19. New England Journal of Medicine, 382(21), 2049-2055.

Etheridge B., \& Spanting L. (2020). The gender gap in mental well-being during the Covid-19 outbreak: Evidence from the UK. https://www.iser.essex.ac.uk/research/publications/working-papers/iser/2020-08.pdf.

Everly, G. S., \& Lating, J. M. (2013). Resilience: The Final Frontier. In: A Clinical Guide to the Treatment of the Human Stress Response. New York: Springer.

Felix, I., A. M., Ilanit Hasson O., \& Giancarlo, D. (2020). Psychological intervention and Covid-19: What we know so far and what we can do. J Contemp Psychother, 1-8

Feldman, D.B., Rand, K.L., \& Kahle-Wrobleski, K. (2009). Hope and goal attainment: Testing a basic explanation of hope theory. Journal of Social and Clinical Psychology, 28(4), 479-497.

Fletcher, R., Kalogeropoulos, A., \& Nielsen, R. K. (2020). News media broadly trusted as source of coronavirus information, views of UK government response highly polarised. Oxford: Reuters Institute for the Study of Journalism.

Folkman, S. (2010). Stress, coping, and hope. Psycho-Oncology, 19(9), 901-908.

Fredrickson, B. L. (2009). Positivity. New York: Crown.

Galanakis, M. M. (2011). Introduction to Positive Psychology (in Greek). Athens: Topos.

Ganie, A. (2020). Children's reactions to lockdown: Need of nurturing resilience in children exposed to Covid-19 pandemic: A review. International Journal of Research in Social Sciences, 10, 7.

Gianesini, C. (2011). Resilience as a relational construct: Theoretical and empirical evidences (Doctoral Thesis). University of Padua. http://paduaresearch.cab.unipd.it/4432/2/PH_thesis_2012_(in_stampa_24-01-2012).pdf.

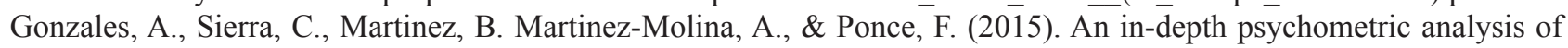
the connor-davidson resilience scale: Calibration with rasch-andrich model. Health Quality Life Outcomes, $13,154$.

Gooding PA., Hurst A., Johnson J., \& Tarrier N. (2012). Psychological resilience in young and older adults. International Journal Geriatric Psychiatry, 27, 262-270.

Hames, J. L., \& Joiner, T. E. (2012). Resiliency factors may differ as a function of self-esteem level: Testing the efficacy of two types of positive self-statements following a laboratory stressor. Journal of Social and Clinical Psychology, 
31(6), 641-662.

Herrman, H., Stewart, D. E., Diaz-Granados, N., Berger, E. L., Jackson, B., \& Yuen, T. (2011). What is resilience? Canadian Journal of Psychiatry, 56(5), 258-265.

Killgore, W., Taylor, E. C., Cloonan, S. A., \& Dailey, N. S. (2020). Psychological resilience during the Covid-19 lockdown. Psychiatry research, 291, 113216.

Kobasa, S.C, Maddi, S.R., \& Kahn, S. (1982). Hardiness and health: A prospective study. Journal of Personality Social Psychology, 42(1), 168-177.

Lopez, S.J., Ciarlelli, R., Coffman, L., Stone, M., \& Wyatt, L. (2000). Diagnosing for strengths: On measuring hope building blocks. Handbook of Hope Theory, Measures, \& Applications, 57-85.

Luthar, S. S. (2000). The construct of resilience: Implications for interventions and social policies. Development and Psychopathology, 12(4), 857-885.

Masten, A. S. (2011). Resilience in children threatened by extreme adversity: Frameworks for research, practice, and translational synergy. Development and Psychopathology, 23(2), 493-506.

McCray, J.A., King, A.R., \& Bailly, M.D. (2005). General and gender-specific attributes of the psychology major. Journal of General Psychology, 132(2), 139-150.

McGinty EE, Rachel Presskreischer, \& Hahrie Han. (2020). Psychological distress and loneliness reported by US adults in 2018 and April 2020. JAMA, 324(1), 93-94.

Mruk, C. (1999). Self-Esteem: Research, Theory and Practice. London: Free Association Books.

Naglieri, J. A., \& LeBuffe P. A. (2005). Measuring resilience in children, from theory to practice. Handbook of Resilience in Children, 107-121.

Nielsen, R. K., Kalogeropoulos, A., \& Fletcher, R. (2020). UK Public Opinion Polarised on News Coverage of Government Coronavirus Response and Concern Over Misinformation. Oxford: Reuters Institute for the Study of Journalism.

Nygren, B., Jonsen, E., \& et al. (2005). Resilience, sense of coherence, purpose in life and self-transcendence in relation to perceived physical and mental health among the oldest old. Aging and Mental Health, 9(4), 354-362.

OCEO. (2020). Youth and Covid-19: Response, recovery and resilience. OECD Policy Responses to Coronavirus (Covid-19), 1-35.

Ong, A.D., Bergeman, C.S., \& Boker, S.M. (2009). Resilience comes of age: Defining features in later adulthood. Journal of Personality, 77(6), 1777-1804.

Raghavan, S. (2020). The relationship between cultural variables and resilience to psychological trauma: A systematic review of the literature. American Psychological Association. https://doi.org/10.1037/trm0000239.

Ryff, C. D, Friedman, E. M., Morozinki, J. A., \& Tsenkova V. (2012). Psychological resilience in adulthood and later life: Implications for health. Annual Review of Gerontology and Geriatrics, 32(1), 72-112.

Shanthakumar, S. G., Seetharam, A., \& Ramesh, A. (2020). Understanding the socio-economic disruption in the United States during COVID-19's early days. In Proceedings of ACM Conference (Conference'17) (pp. 1-5). ACM. https:// arxiv.org/pdf/2004.05451.pdf.

Smith, G. C., \& Hayslip Jr B. (2012). Resilience in adulthood and later life: What does it mean and where are we heading? Annual Review of Gerontology and Geriatrics, 32(1), 3-28.

Snyder, C. R. (1994). The psychology of hope: You can get there from here. New York: Free Press.

Snyder, C. R., Harris, C., Anderson, J. R., Holleran, S. A., Irving, L. M., Sigmon, Yoshinobu, L., Gibb, J., Langelle C., \& Harney, P. (1991). The will and the ways: Development and validation of an individual-differences measure of hope. Journal of Personality and Social Psychology, 60(4), 570-585.

Staudinger, U. M., Marsike, M., \& Baltes, P. B. (1993). Resilience and levels of reserve capacity in later adulthood: Perspectives from lifespan theory. Development and Psychology, 5(4), 541-566.

Wang, C., Pan, R., Wan, X., Tan, Y., Xu, L., Ho, C. S., \& Ho, R. C. (2020). Immediate psychological responses and associated factors during the initial stage of the 2019 coronavirus disease (Covid-19) epidemic among the general population in China. International Journal of Environmental Research and Public Health, 17(5), 1729.

Werner, E. E. (2000). Protective Factors and Individual Resilience. New York: Cambridge University Press.

Wright, M. O., Masten, A. S., \& Narayan, A. J. (2013). Resilience processes in development: Four waves of research on positive adaptation in the context of adversity. Handbook of Resilience in Children, 15-37. https://ink.springer. com/chapter/10.1007\%2F978-1-4614-3661-4_2.

Zhou, X. (2020). Psychological crisis interventions in Sichuan Province during the 2019 novel coronavirus outbreak. Psychiatry Research, 286, 112895. 